\title{
Review
}

\section{Pharmacological actions and therapeutic applications of Salvia miltiorrhiza depside salt and its active components}

\author{
Wen-yu WU, Yi-ping WANG* \\ State Key Laboratory of Drug Research, Shanghai Institute of Materia Medica, Chinese Academy of Sciences, Shanghai 201203, \\ China
}

\begin{abstract}
Salvia miltiorrhiza, a traditional medical herb known as danshen, has been widely used in China to improve blood circulation, relieve blood stasis, and treat coronary heart disease. S miltiorrhiza depside salt is a novel drug recently developed at the Shanghai Institute of Materia Medica; it contains magnesium lithospermate B (MLB) and its analogs, rosmarinic acid (RA) and lithospermic acid (LA), as active components. The drug has been used in the clinic to improve blood circulation and treat coronary heart disease. The pharmacological effects of the depside salt from S miltiorrhiza and its components have been extensively investigated. Experimental studies have demonstrated that magnesium lithospermate B possesses a variety of biological activities, especially protective effects in the cardiovascular system such as attenuation of atherosclerosis and protection against myocardial ischemia-reperfusion injury. Rosmarinic acid and lithospermic acid also show beneficial effects on the cardiovascular system. This paper reviews the recent findings regarding the mechanisms underlying the pharmacological actions of the active components of S miltiorrhiza depside salt, based on published works and our own observations.
\end{abstract}

Keywords: traditional Chinese medicine; Salvia miltiorrhiza depside salt; magnesium lithospermate B (lithospermic acid B or salvianolic acid B); rosmarinic acid; lithospermic acid; cardiovascular activities

Acta Pharmacologica Sinica (2012) 33: 1119-1130; doi: 10.1038/aps.2012.126; published online 3 Sep 2012

\section{Introduction}

The dried roots of Salvia miltiorrhiza, a traditional medical herb known as danshen, are used in China for the treatment of coronary heart disease, hepatitis, menstrual disorders, menostasis, blood circulation diseases, and other cardiovascular diseases $^{[1]}$. The chemical constitutents of $S$ miltiorrhiza have been studied for more than 60 years, but research has been focused mainly on the lipophilic diterpenoid quinones. In recent decades, the pharmacological activities of the water-soluble components of $S$ miltiorrhiza have been investigated, including the active constituents magnesium lithospermate B (MLB, also called salvianolic acid B), rosmarinic acid (RA), lithospermic acid (LA), prolithospermic acid, ammonium potassium lithospermate $\mathrm{B}$, and magnesium salvianolate E oligomers of caffeic acids (Figure 1) ${ }^{[2]}$. Pharmacological studies have shown that water-soluble extracts from danshen can provide an alternative regimen for the prevention of ischemic heart disease ${ }^{[3]}$.

\footnotetext{
* To whom correspondence should be addressed.

E-mail ypwang@mail.shcnc.ac.cn

Received 2012-07-16 Accepted 2012-08-01
}

In 2005, the State Food and Drug Administration (SFDA) of China approved a new drug application for $S$ miltiorrhiza depside salt, with MLB, RA, and LA as the primary active compounds, for the treatment of chronic angina. A clinical study showed that intravenous infusion of $S$ miltiorrhiza depside salt had an observable therapeutic effect in patients with the coronary heart disease angina pectoris ${ }^{[4]}$. In this article, we review the recent findings from our group and others in order to present the pharmacological profiles and therapeutic applications of the main components of $S$ miltiorrhiza depside salt - MLB, RA, and LA - and the mechanisms underlying their clinical efficacy.

\section{Pharmacokinetic properties of S miltiorrhiza depside salt} An understanding of the pharmacokinetics and bioavailability of herbal medicinal products can enable us to link data from pharmacological assays to clinical effects and also help in designing rational dosage regimens. The pharmacokinetic properties of $S$ miltiorrhiza depside salt have been investigated using liquid chromatography-tandem mass spectrometry following intravenous administration in animals ${ }^{[5,6]}$ and healthy 

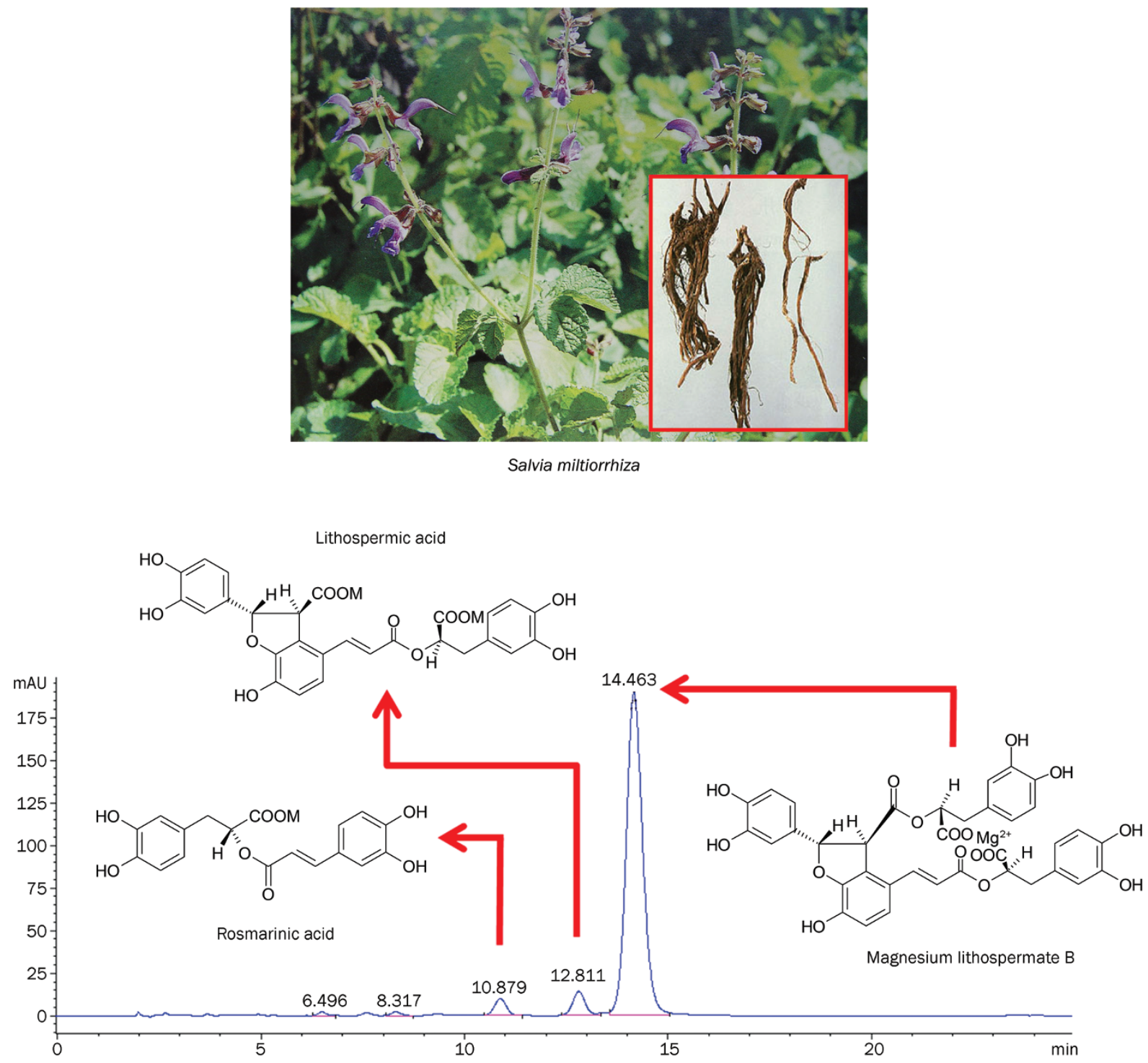

Figure 1. Salvia miltiorrhiza depside salt and its main components.

volunteers $^{[7]}$. The bioavailability of MLB is extremely low; it has been calculated to be only $1.07 \% \pm 0.43 \%$ in $\operatorname{dogs}^{[8]}$ and $0.02 \%$ in rats ${ }^{[9]}$. The elimination time $\left(t_{1 / 2}\right)$ of MLB, RA, and LA is $1.04,0.75$, and $2.0 \mathrm{~h}$, respectively, in rats ${ }^{[5]} ; 0.71,0.51$, and $0.83 \mathrm{~h}$ in $\operatorname{dogs}^{[6]}$; and 2.33, 0.23, and $3.74 \mathrm{~h}$ in healthy Chinese volunteers ${ }^{[7]}$, following intravenous administration of $S$ miltiorrhiza depside salt. The pharmacokinetic properties of $S$ miltiorrhiza depside salt are summarized in Table 1. Given that MLB has been shown to have low permeability through Caco-2 cell monolayers, its low bioavailability could be due to poor absorption and metabolism ${ }^{[10]}$. Overall, extensive metabolism, including a first-pass effect, poor absorption, and wide distribution contributed significantly to MLB's extremely low systemic bioavailability ${ }^{[9]}$.

Cytochrome P450 isoenzymes (CYPs), the most important phase I enzymes in the metabolism of xenobiotics, are involved in the metabolism of most drugs. Recently, MLB was found to act as a weak inhibitor of CYP1A2 in human liver microsomes ${ }^{[11]}$, to down-regulate CYP3A4 and CYP1A2 mRNA expression in the absence of rifampicin and to inhibit rifampicin-induced CYP3A4 mRNA expression in HepG2 cells $^{[12]}$. However, Liu et al reported that MLB could significantly transactivate the CYP3A4 reporter gene construct in either HepG2 or Huh7 cells and the PXR mRNA expression in LS174T cells ${ }^{[13]}$. These data suggest that MLB may modulate the metabolism of the other drugs by induction or inhibition of specific drug-metabolizing enzymes. Additionally, the metabolism of MLB itself can be changed.

\section{Pharmacological actions of magnesium lithospermate B (MLB)}

Salvianolic acid B and lithospermic acid B were identified 
Table 1. Pharmacokinetic properties of S miltiorrhiza depside salt.

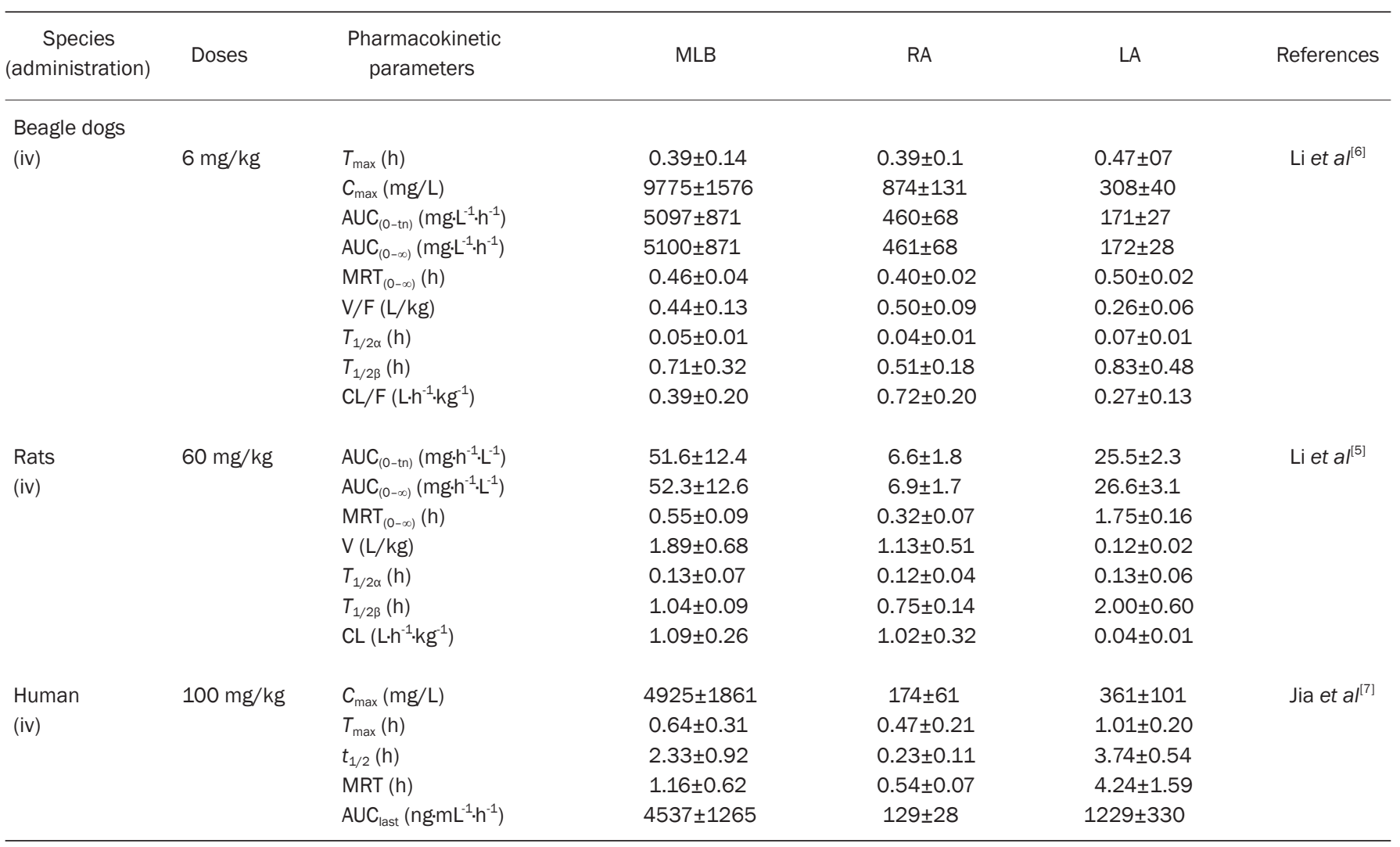

$\mathrm{Ae}_{0-24 \mathrm{~h}}$, excreted in 24-h urine sample as unchanged drug; AUC, area under the plasma level-time curve; $C_{\max }$, maximum concentration of drug; $\mathrm{CL}$, systemic clearance; MRT, mean retention time; NC, not calculable; SD rat, Sprague-Dawley rat; $t_{1 / 2}$, terminal elimination half-life; $T_{1 / 2 \alpha}$, absorption halflife; $T_{1 / 2 \beta}$, elimination half-life; $T_{\max }$, time of occurrence for maximum (peak) drug concentration.

decades ago as the major components of danshen. They were originally reported to have identical structures except for the configurational assignments of two stereocenters, recently through chemical correlation, they were shown to be the same compound $^{[14]}$.

Currently, MLB is used as a quality-control ingredient and active marker for danshen products by the National Pharmacopoeia Council of China ${ }^{[15]}$. As the major component (content $>85 \%$ ) of $S$ miltiorrhiza depside salt, the pharmacological actions of MLB have been extensively investigated.

\section{Attenuation of atherosclerosis}

A large body of evidence has demonstrated that MLB is capable of preventing the development of atherosclerosis in vivo and in vitro. The in vivo evidence for the anti-atherosclerotic effects of MLB is compiled in Table 2. Intimal hyperplasia results from the proliferation and migration of vascular smooth muscle cells (VSMCs) after endothelial injury and excessive oxidative stress, which were significantly reduced by MLB treatment. It has been found that PDGF-BB, SDF-1a, and high glucose could induce VSMC proliferation and migration, which were suppressed by MLB through the following signaling pathways: those induced by PDGF-BB was mediated via inhibiting the phosphorylation of PI3K/Akt and ERK ${ }^{[16]}$, those induced by SDF-1a via suppressing the expression levels of CXCR4 receptor and downstream molecules of SDF-1a/ CXCR4 axis ${ }^{[17]}$, and those induced by high glucose via inducing the nuclear factor erythroid 2-related factor-2 (Nrf2)antioxidant responsive element (ARE)-NAD(P)H:quinone oxidoreductase-1 (NQO1) (Nrf2-ARE-NQO1) pathway ${ }^{[18]}$. Furthermore, MLB was able to induce VSMC apoptosis by up-regulating $\mathrm{p} 53^{[19]}$. Most recently, Cho et al elucidated the mechanisms by which MLB regulates the cellular proliferation in VSMCs. Using fluorescein isothiocyanate (FITC)conjugated MLB to track its cellular localization, these authors found that MLB bound to the non-muscle myosin heavy chain (NMHC-IIA), thereby allowing MLB to suppress the PDGFinduced proliferation of VSMCs ${ }^{[20]}$. In addition to inhibiting the proliferation and migration of VSMCs, subsequently preventing neointimal hyperplasia, many other effects are involved in the anti-atherogenic effects of MLB, including the scavenging of ROS/free radicals, attenuation of injury of the vascular endothelium, inhibition of inflammatory reactions, avoidance of lipid deposition, and modulation of the immune response. 
Table 2. Anti-atherosclerotic effects of magnesium lithospermate B.

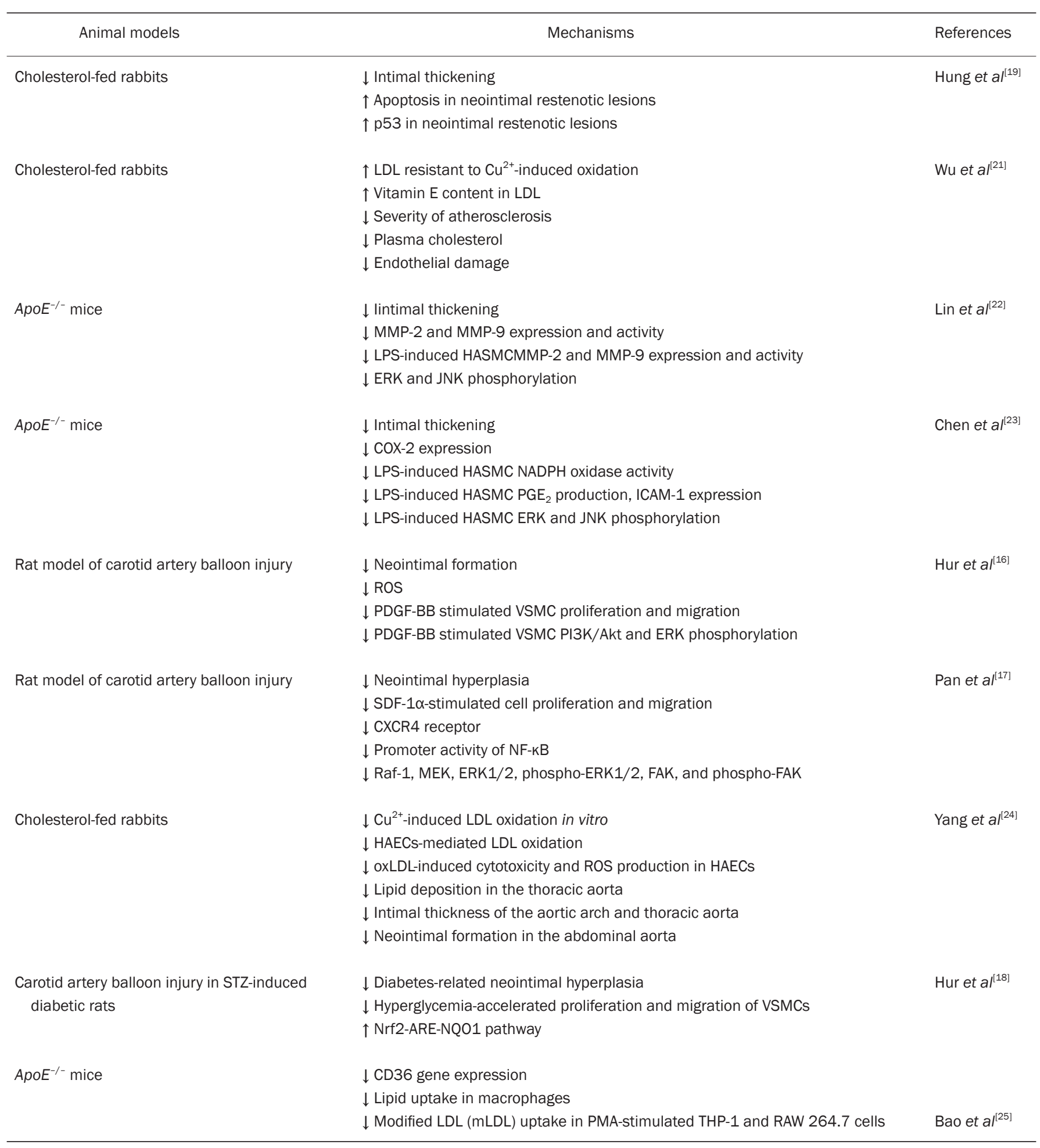

COX-2, cyclooxygenase-2; CXCR4 receptor, cysteine-x-cysteine chemokine receptor 4; HAECs, human aortic endothelial cells; LDL, low-density lipoprotein; HASMC, human vascular smooth muscle cell; ICAM-1, intercellular adhesion molecule 1; LPS, lipopolysaccharide; MMP, matrix metalloproteinase; Nrf2ARE-NQ01, nuclear factor erythroid 2-related factor-2 (Nrf2)-antioxidant responsive element (ARE)-NAD(P)H:quinone oxidoreductase-1 (NQO1); oxLDL, oxidized LDL; PDGF, platelet-derived growth factor; PMA, phorbol-12-myristate-13-acetate; ROS, reactive oxygen species; VSMC, vascular smooth muscle cell; STZ, streptozotocin. 


\section{Free radical scavenging and anti-oxidant activity}

MLB exhibited iron chelating and scavenging activities against free hydroxyl radicals (HO·), superoxide anion radicals $\left(\mathrm{O}_{2}{ }^{-}\right)$, 1,1-diphenyl-2-picryl-hydrazyl (DPPH) radicals, 2-azinobis(3-ethylbenzthiazoline-6-sulfonic acid (ABTS) radicals and hydrogen peroxide $\left(\mathrm{H}_{2} \mathrm{O}_{2}\right)^{[26]}$, as well as xanthine oxidase inhibitory activity ${ }^{[27]}$. Intracellular and extracellular oxidative stress can induce oxidative modification of low-density lipoprotein (LDL) to oxLDL, one of the important substances that drive intimal immune cell infiltration. Inhibiting the formation of oxLDL is beneficial in preventing the development of atherosclerosis ${ }^{[28]}$. It was found that MLB inhibited serum LDL oxidation ${ }^{[24]}$, and MLB-treated LDL exhibited vitamin E-binding ability and was resistant to $\mathrm{Cu}^{2+}$-induced oxidation $^{[21]}$. These studies suggest that MLB can attenuate pathological increases in the peroxidation of lipids, thus suppressing the development and progression of atherosclerosis. Consistent with this line of evidence, our previous study showed that MLB prevented auto-oxidation and $\mathrm{Fe}^{2+} / \mathrm{VitC}$ induced lipid peroxidation in rat serum, liver, kidney, heart, and brain homogenates in vitro and exerted similar effects in an ex vivo experiment with the exception of brain homogenate ${ }^{[29]}$. In addition to preventing lipid oxidation, the scavenging of ROS contributes to the effects of MLB on preventing injury-induced neointimal formation in rats and in cholesterol-fed rabbits, inhibiting VSMC proliferation and migration, and preventing human aortic endothelial cells from oxidative injury-mediated cell death ${ }^{[16,24]}$.

It has been shown that MLB suppresses NADPH oxidase activity, subsequently reducing ROS generation in response to TNF- $\mathrm{a}, \mathrm{H}_{2} \mathrm{O}_{2}$, and Ang-II in human aortic smooth muscle cells (HASMCs) ${ }^{[30]}$; MLB has also been shown to directly reduce excessive ROS generated by high glucose through the enhancement of high glucose-induced Nrf2 action and the subsequent heme oxygenase-1 expression in HEK293T cells ${ }^{[31]}$. Moreover, the entire Nrf2-ARE signaling pathway has been found to be involved in the antioxidative effects of MLB. In that study, MLB acted at least in part by activating the Nrf2ARE-NQO1 pathway and also restored redox balance during hyperglycemia-induced chronic oxidative stress ${ }^{[18]}$. Thus, we believe that the prevention of oxidative stress and related vascular complications by MLB contributes to its anti-atherosclerotic effects.

\section{Preventing endothelial dysfunction}

The death or injury of endothelial cells (ECs) may contribute to the initial endothelial pathophysiological processes, such as angiogenesis, atherosclerosis, and thrombosis. MLB was found to attenuate the endothelial damage in cholesterol-fed rabbits $^{[21]}$ and to protect human endothelial cells from oxidative stress-induced damage via inducing the expression of glucose-regulated protein 78 (GRP78) ${ }^{[32]}$, thus suggesting that MLB could maintain the integrity of the initial endothelium. Endothelial injury leads to a significant increase in LDL permeability, which plays a role in the formation and development of atherosclerosis. MLB inhibited the VEGF-induced
LDL permeability of $\mathrm{ECs}^{[33]}$ and reduced the TNF-a-induced permeability and disorganization of vascular endothelialcadherin in ECs by decreasing VEGF protein expression via modulation of the ERK pathway ${ }^{[34]}$. Loss of the cell-cell adherence junction also increases endothelial permeability. MLB could attenuate TNF-a-induced tyrosine phosphorylation of junctional proteins, including vascular endothelial cadherin and $\beta$-catenin. An immunoprecipitation study showed that MLB prevented $\beta$-catenin disassociation from the cytoskeleton in TNF-a-treated HUVECs ${ }^{[35]}$. In addition to reducing the endothelial permeability, MLB also modulated the hemostatic properties of ECs. MLB increased the fibrinolytic and anticoagulant potential of cultured HUVECs by up-regulating the expression of tissue-type plasminogen activator (t-PA) and thrombomodulin (TM) and down-regulating the expression of plasminogen activator inhibitor type 1 (PAI- 1$)^{[36]}$. The NF-kB and ERK-AP-1 pathways were considered possible targets of MLB in the attenuation of the PAI-1 production response to TNF- $a$ in HUVECs ${ }^{[37]}$.

Atherogenic recruitment of leukocytes involves a sequence of rolling, firm adhesion, lateral migration and transendothelial diapedesis and is controlled by chemokines. During atherosclerosis, circulating monocytes and lymphocytes may interact with adhesion molecules, such as vascular adhesion molecule-1 (VCAM-1), intercellular adhesion molecule-1 (ICAM-1) and E-selectin on ECs, to establish firm adhesion, which may be an early event in atherogenesis. MLB pretreatment notably attenuated TNF-a-induced expression of VCAM-1 and ICAM-1 and the binding of monocytes to HAECs, which was associated with its anti-inflammatory property through inhibition of the NF-kB pathway ${ }^{[38,39]}$. In IFN- $\gamma$-treated ECs, MLB inhibited IFN $\gamma$-induced JAK-STAT signaling pathways and consequently suppressed IFN- $\gamma-$ induced expression of chemokines (including IP-10, Mig, and I-TAC), IP-10 promoter activity, IP-10 protein release, and monocyte adhesion to $\mathrm{ECs}^{[40]}$. These studies support the potential clinical application of MLB in vascular inflammatory diseases, including atherosclerosis.

MLB regulates vascular homeostasis by exerting a number of vasoprotective effects, including the stimulation of vasodilation, suppression of VSMC proliferation, and inhibition of inflammatory responses. Many of these effects are mediated by the most potent endogenous vasodilator, NO. Endothelial cell-derived NO acts as an important mediator in the cardiovascular, nervous, and immune systems ${ }^{[41]}$. Our previous studies showed that MLB could inhibit $\mathrm{Ca}^{2+}$ influx and stimulate NO production in ECs treated with hypoxia/ reoxygenation, which in turn could attenuate cell injury ${ }^{[42]}$. MLB could also inhibit $\mathrm{Ca}^{2+}$ influx and decrease $\mathrm{NO}$ release in ECs exposed to hypoxia and attenuate cell injury in $\mathrm{ECs}^{[43]}$. In HUVECs, MLB enhanced NO production via the AMPK/ PI3K/Akt pathway ${ }^{[44]}$. MLB was also found to produce endothelium-dependent vasodilation by regulating NO production through the modulation of heme oxygenase- 1 and arginase activities $^{[41]}$.

Taking all these data together, we conclude that MLB dimin- 
ishes endothelial dysfunction through up-regulating antiinflammatory responses and promoting vasodilation, which may contribute to the prevention and treatment of various cardiovascular disorders, including atherosclerosis.

\section{Anti-inflammatory effects and regulation of matrix metallopro- teinases expression and activity}

The inflammatory response is involved in the pathogenesis of atherosclerosis, and the initial degradation of the extracellular matrix (ECM) is an inevitable step for vascular cell hypertrophy, proliferation, and migration, which in turn plays an important role in vascular remodeling and contributes to the vulnerability of atherosclerotic plaques to rupture. Vascular cells, including SMCs, can secrete matrix metalloproteinases (MMPs), the enzymes that selectively digest the individual components of the ECM. MLB treatment effectively attenuated MMP-2, MMP-9 $9^{[22]}$, and COX-2 ${ }^{[23]}$ protein expression in cholesterol-fed $\mathrm{ApoE}^{-/-}$mice, which was related to the reduced thickness of the intima and protection of these mice against atherosclerosis. In HASMCs, MLB reduced the LPS-induced MMP-2 and MMP-9 expression via downregulating the JNK and ERK signaling pathways ${ }^{[22]}$ and inhibited the LPS-induced COX-2 expression via reducing $\mathrm{PGE}_{2}$ production, ICAM-1 expression and NADPH oxidase activity ${ }^{[23]}$. Furthermore, MLB also inhibited MMP-2 expression and activity in response to TNF- $\alpha$, Ang II, and $\mathrm{H}_{2} \mathrm{O}_{2}$ in HASMCs via reducing NADPH oxidase-dependent ROS generation ${ }^{[30]}$. Moreover, MLB downregulated the SDF-1a-stimulated up-regulation of CXCR4 (total and cell-surface levels), Raf-1, FAK, and phospho-FAK, as well as the promoter activity of NF-KB, which provided a beneficial effect against the counteracting effects of inflammation on VSMCs ${ }^{[17]}$. These studies demonstrate that MLB can stabilize arterial atherosclerotic plaques and reduce the risk of coronary heart disease.

\section{Modulation of lipid profiles}

In cholesterol-fed rabbits, MLB treatment attenuated the increase in plasma cholesterol predominantly in $\beta$-VLDL ${ }^{[21]}$. MLB treatment was also found to decrease the atherosclerotic area, cholesterol deposition ${ }^{[21,24]}$ and lipid levels of aortic vessels $^{[25]}$. However, MLB does not exert obvious lipid-lowering effects. The relationship between lipid lowering and the atheroprotective effects of MLB is still unclear. Because dyslipidemia is one of the main risk factors that lead to atherosclerosis, further investigations are required to explore the effects of MLB on cholesterol metabolism.

\section{Potential immunomodulators}

The activation of $\mathrm{T}$ lymphocytes plays a promoting role in the inflammatory processes of atherosclerotic diseases, and functional, immune-stimulating dendritic cells (DCs) have recently been detected in the aortic intima, the site of origin for atherosclerosis. Immunosuppressive methotrexate for treatment of atherosclerosis is currently under investigation ${ }^{[28]}$. One study found that MLB effectively suppressed maturation of human monocyte-derived dendritic cells (h-monDC) induced by ox-
LDL through activation of PPAR $\gamma^{[45]}$ and inhibition of IL-2, IL-4, TNF- $a$, and IFN- $\gamma$ production from activated T cells ${ }^{[46]}$; MLB also effectively reduced the expression of $\mathrm{T}$ cell activation markers CD25 and CD69 ${ }^{[46]}$. With molecular modeling, MLB was found to act as an inhibitor of protein-protein interactions between the SH2 domains of the Src-family kinases, Src and Lck. The potency of MLB binding to Src and Lck was higher than RA, a natural compound known as the Lck SH2 domain inhibitor ${ }^{[47]}$. Because Lck is a T cell-restricted Src family protein tyrosine kinase, and inhibition of the Lck SH2 domain has been suggested as a possible mechanism underlying the immunosuppressant activity of RA, it appears that MLB may act as an immunosuppressive agent ${ }^{[48]}$.

CD36, a member of the class B scavenger receptors, is a highaffinity receptor for oxidatively modified low-density lipoprotein (oxLDL) and has been implicated in the pathogenesis of a variety of vascular inflammatory diseases. MLB was found to be antagonistic against CD36-oxLDL binding, which was further validated by its inhibition of oxLDL uptake in RAW 264.7 cells ${ }^{[49]}$. Yi et al confirmed the specificity and efficacy of MLB in inhibition of CD36-mediated lipid uptake in vitro and in vivo and demonstrated that MLB was an effective CD36 antagonist $^{[25]}$. These results support a role for MLB as an immune modulator with cardioprotective effects, which would increase its therapeutic potential in atherosclerotic pathologies.

\section{Protecting against myocardial ischemia and reperfusion (I/R) injury}

Cardiac glycosides are drugs that are clinically used to relieve the symptoms of congestive heart failure via their reversible inhibition on $\mathrm{Na}^{+} / \mathrm{K}^{+}$-ATPase located in human myocardium. However, narrow safety margins and severe side effects make administration of these drugs difficult. MLB is a non-steroid compound possessing inhibitory activity against $\mathrm{Na}^{+} / \mathrm{K}^{+}-$ ATPase with potency comparable to that of cardiac glycosides but without the apparent adverse effects. Therefore, MLB has great potential in the treatment of congestive heart failure, provided that it undergoes the necessary clinical trials ${ }^{[50]}$. MLB has been found to exert protective effects on the heart in various animal models. In a porcine model of myocardial I/R, MLB increased capillary density and decreased infarct size ${ }^{[51]}$. In C57 mice, MLB was found to inhibit cardiac hypertrophy and infarction and to improve cardiac function at 4 weeks after induction of the infarction ${ }^{[44]}$. In rats with left anterior descending coronary artery ligation, MLB treatment effectively improved left ventricle (LV) function and the appearance of the myocardium as compared with a group with acute myocardial infarction (AMI) ${ }^{[52,53]}$; MLB treatment also prevented myocardial remodeling, a deleterious consequence of myocardial infarction (MI), by significantly down-regulating the mRNA expression level and activity of MMP-9 ${ }^{[52]}$. MLB was found to bind to MMP-9 at the catalytic domain and to function as a competitive inhibitor of MMP- $9^{[54]}$ and thus could attenuate the enhancing effects of MMP-9 on migration, proliferation, collagen synthesis, cytokine secretion, as well as the association between cardiac fibroblasts and myofibro- 
blast transition ${ }^{[55]}$. Metabolomics offers a new approach to the research of traditional Chinese medicines. Lu et al, who analyzed plasma from MI rats and built partial least-squares discriminate analysis (PLS-DA) models, found that MLB was able to regulate 22 identified MI biomarkers in rat plasma ${ }^{[56]}$; this biomarker pattern was similar to the metabolomic profile of propanolol, indicating that the two drugs might have similar mechanisms. They further demonstrated that MLB exhibited a protective effect on MI mainly by decreasing the concentration of cAMP and $\mathrm{Ca}^{2+}$ and inhibiting $\mathrm{PKA}^{[57]}$.

The cardioprotection of MLB could benefit from improving myocardial cell function and/or preventing myocardial cell death. MLB seems to have pleiotropic effects and may act on multiple molecular targets to exert its protection effect on cardiomyocytes. We found that MLB reversibly inhibited L-type $\mathrm{Ca}^{2+}$ current $\left(I_{\mathrm{Ca}, \mathrm{L}}\right)$ without significant effects on the fast-inactivating $\mathrm{Na}^{+}$current $\left(I_{\mathrm{Na}}\right)$, delayed rectifier $\mathrm{K}^{+}$current $\left(I_{\mathrm{K}}\right)$ and inward rectifier $\mathrm{K}^{+}$current $\left(I_{\mathrm{K} 1}\right)$ in single ventricular myocytes of adult guinea pigs, suggesting that the voltagedependent $\mathrm{Ca}^{2+}$ antagonistic effects of MLB work together with its antioxidant action for attenuating heart ischemic injury ${ }^{[58]}$. Furthermore, it was found that MLB administration significantly decreased myocardiocyte apoptosis during I/R via interactions with multiple targets, including elevating superoxide dismutase activity, thioredoxin activity and glutathione concentration; reducing malondialdehyde concentration ${ }^{[51]}$; direct/indirect inhibiting stress-activated protein (SAP) kinase activity and nuclear translocation of the active kinase ${ }^{[59]}$; inhibiting the poly (ADP-ribose) polymerase-1 pathway and improving the integrity of mitochondria and nuclei of heart tissue $^{[53]}$. It was also reported that MLB prevented LPS-induced neonatal cardiomyocyte injury through inhibition of the TLR4NF-кB-TNF-a pathway ${ }^{[60]}$ and protected against cardiotoxicity of doxorubicin in mice through blockade of oxidative stress ${ }^{[61]}$. More recently, MLB was found to protect starving cardiomyocytes by blocking the early stage of autophagic flux and inhibiting the apoptosis that occurred during autophagy ${ }^{[62]}$.

In addition to exerting direct benefit effects on a heart undergoing I/R, MLB can protect the heart from I/R injury through indirect effects, as described below.

First, MLB attenuates the risk of I/ R via anti-atherosclerotic properties (see the section "Attenuation of atherosclerosis").

Second, MLB produces vasodilator and vasorelaxant effects. Studies from our group and those of others have demonstrated that these effects result from attenuating intracellular $\mathrm{Ca}^{2+}$ concentrations $\left(\left[\mathrm{Ca}^{2+}\right]_{\mathrm{i}}\right)$ in VSMCs ${ }^{[63]}$, inhibiting $\mathrm{Ca}^{2+}$ channels in the VSMCs with a minor component mediated by the opening of $\mathrm{K}^{+}$channels ${ }^{[64]}$, decreasing $\left[\mathrm{Ca}^{2+}\right]_{\mathrm{i}}$ by inhibiting $\mathrm{K}^{+}$ currents and depolarizing membrane potential in $\mathrm{ECs}^{[65]}$, activating large-conductance $\mathrm{Ca}^{2+}$-activated $\mathrm{K}^{+}\left(\mathrm{BK}_{\mathrm{Ca}}\right)$ currents in smooth muscle cells ${ }^{[66,67]}$, and inhibiting $\mathrm{K}_{\mathrm{V}}$ currents channels in smooth muscle cells and increasing NO release from endothelium $^{[44,67]}$.

Third, MLB produces antiplatelet, anticoagulant and antithrombotic effects. In myocardial ischemic rabbits, MLB significantly reduced whole-blood and plasma viscosity, improved hemorheology, prevented angiospasm and platelet aggregation, and reduced oxidative injury ${ }^{[68]}$. MLB was also found to delay thrombus-initiation time and damp photochemical reaction-inducedmast cell degranulation in rat mesentery $^{[69]}$. Our previous study showed that MLB decreased the thrombin-activated release of 5-HT and aggregation in rabbit platelets, probably by attenuating $\left[\mathrm{Ca}^{2+}\right]_{i}^{[70]}$. MLB also inhibited platelet aggregation induced by high shear stress ${ }^{[71]}$. Wu et al attributed the antiplatelet effect of MLB to a specific interaction with the platelet collagen receptor $\alpha 2 \beta 1^{[72]}$. Ma et al further demonstrated that the binding of MLB to integrin $\alpha 2 \beta 1$ caused changes in $\left[\mathrm{Ca}^{2+}\right]_{i}$, the levels of cytoskeleton-related proteins such as coronin-1B and the cytoskeletal structure of platelets, and therefore concluded that integrin a $2 \beta 1$ might be one of the direct target proteins of MLB in platelets ${ }^{[73]}$.

Fourth, MLB improved myocardial microperfusion ${ }^{[74]}$, myocardial microvascular reflow ${ }^{[51]}$, as well as coronary blood flow $^{[68]}$. MLB also possessed antihypertensive effects partly due to inhibiting angiotensin converting enzyme activity ${ }^{[75]}$.

Finally, MLB protected bone marrow stem cells from apoptosis $^{[76]}$ and synergized with vitamin $C$ in inducing embryonic stem cell differentiation into matured and functional cardiomyocytes $^{[77]}$.

\section{Other pharmacological actions}

In addition to its effects on the cardiovascular system, MLB has other pharmacological actions as discussed below.

\section{Preventing cerebral ischemia-reperfusion injury and neuro- degeneration}

Cerebral ischemia-reperfusion (I/R) injury is the main reason for the loss of neurons in ischemic cerebrovascular disease. In focal cerebral I/ $\mathrm{R}$ rats, MLB treatment protected the brain against I/ R injury by reducing lipid peroxides, scavenging free radicals and improving energy metabolism ${ }^{[78]}$. MLB exerted neuroprotection against ischemic stroke by inhibiting the $\mathrm{Na}^{+}$/ $\mathrm{K}^{+}$-ATPase via binding to the extracellular pocket of the $\mathrm{Na}^{+}$/ $\mathrm{K}^{+}$-ATPase a subunit and then promoting blood circulation ${ }^{[79]}$. The anti-apoptotic effect of MLB on rheologically induced endothelial injury probably also contributes to its effectiveness in the treatment of cerebrovascular diseases ${ }^{[80]}$.

Alzheimer's disease (AD) and Parkinson's disease (PD) are common degenerative brain disorders. One of the major pathological features of AD is the appearance of senile plaques characterized by extracellular aggregation of amyloid $\beta$-peptide $(A \beta)$ fibrils. Inhibition of $A \beta$ fibril aggregation is therefore regarded as one possible method to halt the progression of AD. MLB was found to inhibit fibril aggregation as well as to destabilize the preformed $\mathrm{A} \beta$ fibrils. Moreover, preincubation with MLB significantly reduced the cytotoxic effect of $A \beta_{1-42}$ on human neuroblastoma SH-SY5Y cells ${ }^{[81]}$. Interestingly, MLB was found to alleviate the memory impairments induced by cholinergic dysfunction or $A \beta_{25-35}$ peptide owing to its antagonism of $\mathrm{GABA}_{\mathrm{A}}$ receptors ${ }^{[22]}$. MLB was also found to protect rat cerebral microvascular endothelial cells (rCMECs) against $\mathrm{H}_{2} \mathrm{O}_{2}$-induced apoptosis through the PI3K/ 
Akt/Raf/MEK/ERK pathway, which may partly contribute to its beneficial effects on $\mathrm{AD}^{[83]}$, given that rCMECs apoptosis is considered partially responsible for the pathogenesis of $\mathrm{AD}$.

PD is associated with mitochondrial dysfunction, oxidative stress, and activation of the apoptotic cascade. MLB was found to exert protective effects on $\mathrm{MPP}^{+}$-induced apoptosis in SH-SY5Y cells (a classic in vitro model for PD) by relieving oxidative stress $^{[84]}$. These observations suggest that MLB has therapeutic potential for the treatment of neurodegenerative diseases.

\section{Inhibitory effects on liver fibrosis}

In a double-blind trial consisting of 60 patients, MLB was found to effectively reverse liver fibrosis in chronic hepatitis B, and it was more effective than the control drug, IFN- $\gamma$, in the reduction of serum HA content, overall decrease of 4 serum fibrotic markers, and decrease of ultrasound imaging score without side effects or toxicity ${ }^{[85]}$. Hepatic fibrosis is characterized by hepatic stellate cell (HSC) activation. It was reported that MLB inhibited HSC proliferation and collagen production, decreased the cells' TGF- $\beta 1$ autocrine levels, ameliorated oxidative damage and eliminated ROS accumulation in hepatocytes ${ }^{[86]}$. MLB was also found to significantly attenuate liver fibrosis and activation of HSCs in thioacetamide-induced hepatic fibrosis in rats ${ }^{[87,88]}$. Taken together, these results suggest that MLB may be used as an effective anti-fibrotic agent in the treatment of hepatic fibrosis.

\section{Improving renal function and preventing diabetic nephropathy}

Tubular epithelial cells can undergo an epithelial-to-mesenchymal transition (EMT), which plays an important role in the pathogenesis of renal interstitial fibrosis (RIF). MLB not only prevented and reversed EMT in HK-2 cells ${ }^{[89,90]}$ but also prevented tubular EMT in $\mathrm{HgCl}_{2}$-induced fibrosis in kidneys ${ }^{[91]}$. Our data showed that MLB ameliorated renal cortical microcirculation $^{[92]}$ and inhibited mesangial cell proliferation ${ }^{[93]}$, which may be related to the drug's renoprotective effects.

Diabetic nephropathy, a common cause of renal disease, accounts for significant morbidity and mortality in patients with diabetes. In in vitro studies, MLB was found to inhibit the high glucose-induced proliferation of mesangial cells and extracellular matrix production, partially through suppressing the cell-cycle process, and also inhibited the activities of MMP-2 and MMP-9 via the NF-kB-dependent pathway ${ }^{[94]}$. MLB also inhibited glucose-induced ROS generation and subsequent PKC inactivation and TGF- $\beta 1$ and fibronectin downregulation in mesangial cells ${ }^{[95]}$. In in vivo studies, MLB treatment effectively inhibited diabetes-associated TGF- $\beta 1$, fibronectin and collagen upregulation in the renal cortex and significantly suppressed the progression of renal injury in streptozotocin-induced diabetic rats (STZR $)^{[95]}$. Similar effects were found in Otsuka Long-Evans-Tokushima Fatty (OLETF) rats with type 2 diabetes ${ }^{[96]}$. The results of these studies suggest that MLB may be a promising therapeutic agent for preventing and treating diabetic nephropathy.

\section{Pharmacological actions of rosmarinic acid (RA)}

Both in vitro and in vivo studies show that RA possesses antioxidant activity as well as anti-inflammatory activities ${ }^{[97,98]}$, which results in the multiple pharmacological actions of RA; this multi-target mechanism is similar to that described previously for MLB.

\section{Pharmacological properties of RA on the cardiovascular system}

First, RA can prevent cardiomyocyte dysfunction. It has been reported that RA could inhibit adriamycin-induced apoptosis in H9c2 cardiac muscle cells by inhibiting ROS generation and JNK and ERK activation ${ }^{[99]}$. RA also prevented cardiopathology and lowered blood pressure in fructose-fed hypertensive rats as a result of inhibition of p22phox NADPH oxidase ${ }^{[100]}$. Second, our results showed that RA possessed a potential vasolilator effect due to decreasing $\left[\mathrm{Ca}^{2+}\right]_{\mathrm{i}}$ in VSMCs. Moreover, RA did not affect the basal level of $\left[\mathrm{Ca}^{2+}\right]_{\mathrm{i}}$ but instead attenuated ATP-stimulated $\left[\mathrm{Ca}^{2+}\right]_{i}$ increases in the absence of external $\mathrm{Ca}^{2+}$ and reduced $\mathrm{KCl}$-induced $\left[\mathrm{Ca}^{2+}\right]_{\mathrm{i}}$ increases in the presence of external $\mathrm{Ca}^{2+[63]}$. Third, RA was found to reduce the risk of MI via its anti-atherosclerotic properties, and it could penetrate membranes to inhibit lipid peroxidation in situ without causing any noticeable alteration of the membrane structure $^{[101]}$. The immunoregulatory activities of RA may also contribute to its anti-atherosclerotic effects. RA was found to induce apoptosis of activated T-cell subsets from rheumatoid arthritis patients via a mitochondrial pathway ${ }^{[102]}$, to inhibit TCR-induced T-cell activation and proliferation ${ }^{[48]}$, and to suppress IFN- $\gamma$-mediated induction of indoleamine 2,3-dioxygenase transcription via down-regulation of STAT1 activation in IFN- $\gamma$-stimulated murine bone marrow-derived dendritic cells ${ }^{[103]}$.

\section{Other pharmacological findings}

In addition to the benefits to the cardiovascular system, RA has other pharmacological effects. (1) Due to its scavenging of peroxynitrite $\left(\mathrm{ONOO}^{-}\right)$, daily consumption of RA exhibited protective effects against the memory impairments caused by the neurotoxicity of $A \beta_{25-35}{ }^{[104]}$. In addition, it has been reported that RA could protect MES23.5 dopaminergic cells against 6-OHDA- ${ }^{[105]}$ or MPP ${ }^{+[106]}$-induced neurotoxicity in vitro and achieve neurorescue effects in 6-ODHA-lesioned rat model of PD in vivo ${ }^{[107]}$. (2) RA has an early renal protective role in nephritic damage. RA was found to be potent in the treatment of diabetic nephropathy. It reduced expression of renal connective-tissue growth factor (CTGF) in STZ-induced rat animal models and in high glucose-stimulated HK-2 cells ${ }^{[108]}$. (3) RA treatment in mice with existing cholestatic liver fibrosis inhibited HSC activation and progression of liver fibrosis via PPARY derepression mediated by suppression of canonical Wnt signaling in $\mathrm{HSCs}^{[109]}$.

\section{Pharmacological actions of lithospermic acid (LA)}

LA is a competitive inhibitor of xanthine oxidase that is able to directly scavenge superoxide and inhibit superoxide pro- 
duction in vitro and thus exhibits hypouricemic and antiinflammatory actions in vivo ${ }^{[110]}$. Our group reported that LA exerted vasodilator action by modulating $\mathrm{Ca}^{2+}$ homeostasis in VSMCs $^{[63]}$ and prevented atherosclerosis by inhibiting VSMC proliferation and migration ${ }^{[111]}$.

\section{Summary and perspectives}

$S$ miltiorrhiza depside salt has been widely prescribed for years. Further investigations to elucidate the mechanisms underlying the protective actions of $S$ miltiorrhiza depside salt and its effects on cardiovascular diseases (CVDs) are under way in our laboratory. The drug comprises three safe and effective components with multiple pharmacological actions, accounting for its pleiotropic pharmacological effects, and it may act at multiple molecular targets, mainly because of its anti-inflammatory and anti-oxidative activities. Currently, $S$ miltiorrhiza depside salt is used primarily for treating CVDs and other circulatory disturbance-related diseases.

Combining $S$ miltiorrhiza depside salt with drugs used to treat hepatic fibrosis (ie, malotilate) may enhance their therapeutic effects. Combining $S$ miltiorrhiza depside salt with antidiabetic drugs reduces the severity of complications of diabetes, such as diabetic nephropathy and diabetic atherosclerosis. However, the possibility that such combinations may result in adverse drug interactions must be taken into account. Targeting cellular functions as a system rather than the single-target level significantly increases therapeutic potency. Further research is warranted to address the mechanisms of the multitarget actions of $S$ miltiorrhiza depside salt and to translate this knowledge into clinical practice.

\section{Acknowledgements}

This work was supported by a grant from the Shanghai Committee of Science and Technology, China (No 09DZ1977402) and the Ministry of Science and Technology of China (No 2010ZX09502-003 and 2003AA2Z3269).

\section{References}

1 Li LN. Biologically active components from traditional Chinese medicines. Pure Appl Chem 1998; 70: 547-54.

2 Zhou CX, Luo HW, Niwa M. Studies on isolation and identification of water-soluble constituents of Salvia miltiorrhiza. J Chin Pharm Univ 1999; 30: 411-6.

3 Sun J, Huang SH, Tan BK, Whiteman M, Zhu YC, Wu YJ, et al. Effects of purified herbal extract of Salvia miltiorrhiza on ischemic rat myocardium after acute myocardial infarction. Life Sci 2005; 76 : 2849-60.

4 Zhang Q, Liu AD, Huang YS. Clinical non-inferiority trial on treatment of coronary heart disease angina pectoris of Xin-blood stasis syndrome type with lyophilized Salvia salt of lithospermic acid powder for injection. Chin J Integr Med 2006; 12: 12-8.

5 Li X, Yu C, Lu Y, Gu Y, Lu J, Xu W, et al. Pharmacokinetics, tissue distribution, metabolism, and excretion of depside salts from Salvia miltiorrhiza in rats. Drug Metab Dispos 2007; 35: 234-9.

6 Li X, Yu C, Sun W, Liu G, Jia J, Wang Y. Simultaneous determination of magnesium lithospermate $B$, rosmarinic acid, and lithospermic acid in beagle dog serum by liquid chromatography/tandem mass spectrometry. Rapid Commun Mass Spectrom 2004; 18: 2878-82.

7 Jia JY, Lu YL, Li XC, Liu GY, Li SJ, Liu Y, et al. Pharmacokinetics of depside salts from Salvia miltiorrhiza in healthy Chinese volunteers: A randomized, open-label, single-dose study. Curr Ther Res 2010; 71: 260-71.

8 Gao DY, Han LM, Zhang LH, Fang XL, Wang JX. Bioavailability of salvianolic acid $B$ and effect on blood viscosities after oral administration of salvianolic acids in beagle dogs. Arch Pharm Res 2009; 32: 773-9.

9 Zhang Y, Akao T, Nakamura N, Duan CL, Hattori M, Yang XW, et al. Extremely low bioavailability of magnesium lithospermate $B$, an active component from Salvia miltiorrhiza, in rat. Planta Med 2004; 70: $138-42$.

$10 \mathrm{Kim} \mathrm{HH}$, Kim J, Ji HY, Kim YC, Sohn DH, Lee BM, et al. Pharmacokinetics of lithospermic acid B isolated from Salvia miltiorrhiza in rats. J Toxicol Environ Health A 2005; 68: 2239-47.

11 Qiu F, Zhang R, Sun J, Jiye A, Hao H, Peng Y, et al. Inhibitory effects of seven components of danshen extract on catalytic activity of cytochrome P450 enzyme in human liver microsomes. Drug Metab Dispos 2008; 36: 1308-14.

12 Wang QL, Wu Q, Tao YY, Liu CH, El-Nezami H. Salvianolic acid B modulates the expression of drug-metabolizing enzymes in HepG2 cells. Hepatobiliary Pancreat Dis Int 2011; 10: 502-8.

13 Liu YH, Mo SL, Bi HC, Hu BF, Li CG, Wang YT, et al. Regulation of human pregnane $X$ receptor and its target gene cytochrome P450 3A4 by Chinese herbal compounds and a molecular docking study. Xenobiotica 2011; 41: 259-80.

14 Watzke A, O'Malley SJ, Bergman RG, Ellman JA. Reassignment of the configuration of salvianolic acid $B$ and establishment of its identity with lithospermic acid B. J Nat Prod 2006; 69: 1231-3.

15 Zhao Y, Hao Y, Ji H, Fang Y, Guo Y, Sha W, et al. Combination effects of salvianolic acid $B$ with low-dose celecoxib on inhibition of head and neck squamous cell carcinoma growth in vitro and in vivo. Cancer Prev Res (Phila) 2010; 3: 787-96.

16 Hur KY, Seo HJ, Kang ES, Kim SH, Song S, Kim EH, et al. Therapeutic effect of magnesium lithospermate $B$ on neointimal formation after balloon-induced vascular injury. Eur J Pharmacol 2008; 586: 22633.

17 Pan $\mathrm{CH}$, Chen CW, Sheu MJ, Wu CH. Salvianolic acid B inhibits SDF1alpha-stimulated cell proliferation and migration of vascular smooth muscle cells by suppressing CXCR4 receptor. Vascul Pharmacol 2012; 56: 98-105.

18 Hur KY, Kim SH, Choi MA, Williams DR, Lee YH, Kang SW, et al. Protective effects of magnesium lithospermate $B$ against diabetic atherosclerosis via Nrf2-ARE-NQ01 transcriptional pathway. Atherosclerosis 2010; 211: 69-76.

19 Hung HH, Chen YL, Lin SJ, Yang SP, Shih CC, Shiao MS, et al. A salvianolic acid B-rich fraction of Salvia miltiorrhiza induces neointimal cell apoptosis in rabbit angioplasty model. Histol Histopathol 2001; 16: 175-83.

20 Cho YH, Lim EY, Kim JM, Jung M, Lee HC, Seo M, et al. Nonmuscle myosin heavy chain and histone $\mathrm{H} 3$ are intracellular binding partners of lithospermic acid B and mediate its antiproliferative effect on VSMCs. Curr Med Chem 2012; 19: 1731-7.

21 Wu YJ, Hong CY, Lin SJ, Wu P, Shiao MS. Increase of Vitamin E Content in LDL and reduction of atherosclerosis in cholesterolfed rabbits by a water-soluble antioxidant-rich fraction of Salvia miltiorrhiza. Arterioscler Thromb Vasc Biol 1998; 18: 481-6.

22 Lin SJ, Lee IT, Chen YH, Lin FY, Sheu LM, Ku HH, et al. Salvianolic acid $B$ attenuates MMP-2 and MMP-9 expression in vivo in apolipoprotein-E-deficient mouse aorta and in vitro in LPS-treated human 
aortic smooth muscle cells. J Cell Biochem 2007; 100: 372-84.

23 Chen YL, Hu CS, Lin FY, Chen YH, Sheu LM, Ku HH, et al. Salvianolic acid $\mathrm{B}$ attenuates cyclooxygenase-2 expression in vitro in LPS-treated human aortic smooth muscle cells and in vivo in the apolipoproteinE-deficient mouse aorta. J Cell Biochem 2006; 98: 618-31.

24 Yang TL, Lin FY, Chen YH, Chiu JJ, Shiao MS, Tsai CS, et al. Salvianolic acid B inhibits low-density lipoprotein oxidation and neointimal hyperplasia in endothelium-denuded hypercholesterolaemic rabbits. J Sci Food Agric 2011; 91: 134-41.

25 Bao Y, Wang L, Xu Y, Yang Y, Wang L, Si S, et al. Salvianolic acid B inhibits macrophage uptake of modified low density lipoprotein ( $\mathrm{mLDL}$ ) in a scavenger receptor CD36-dependent manner. Atherosclerosis 2012; 223: 152-9.

26 Zhao GR, Zhang HM, Ye TX, Xiang ZJ, Yuan YJ, Guo ZX, et al. Characterization of the radical scavenging and antioxidant activities of danshensu and salvianolic acid B. Food Chem Toxicol 2008; 46: 73-81.

27 Liu X, Chen R, Shang Y, Jiao B, Huang C. Superoxide radicals scavenging and xanthine oxidase inhibitory activity of magnesium lithospermate B from Salvia miltiorrhiza. J Enzyme Inhib Med Chem 2009; 24: 663-8.

28 Weber C, Noels $\mathrm{H}$. Atherosclerosis: current pathogenesis and therapeutic options. Nat Med 2011; 17: 1410-22.

29 Wu XJ, Wang YP, Wang W, Sun WK, Xu YM, Xuan LJ. Free radical scavenging and inhibition of lipid peroxidation by magnesium lithospermate B. Acta Pharmacol Sin 2000; 21: 855-8.

30 Zhang HS, Wang SQ. Salvianolic acid B from Salvia miltiorrhiza inhibits tumor necrosis factor-alpha (TNF-alpha)-induced MMP-2 upregulation in human aortic smooth muscle cells via suppression of $\mathrm{NAD}(\mathrm{P}) \mathrm{H}$ oxidase-derived reactive oxygen species. J Mol Cell Cardiol 2006; 41: 138-48.

31 Qu J, Ren X, Hou RY, Dai XP, Zhao YC, Xu XJ, et al. The protective effect of magnesium lithospermate $B$ against glucose-induced intracellular oxidative damage. Biochem Biophys Res Commun 2011; 411: 32-9.

32 Wu HL, Li YH, Lin YH, Wang R, Li YB, Tie L, et al. Salvianolic acid B protects human endothelial cells from oxidative stress damage: $a$ possible protective role of glucose-regulated protein 78 induction. Cardiovasc Res 2009; 81: 148-58.

33 Qui Y, Rui YC, Zhang L, Li TJ, Zhang WD. VEGF induced hyperpermeability in bovine aortic endothelial cell and inhibitory effect of salvianolic acid B. Acta Pharmacol Sin 2001; 22: 117-20.

34 Ding M, Ye TX, Zhao GR, Yuan YJ, Guo ZX. Aqueous extract of Salvia miltiorrhiza attenuates increased endothelial permeability induced by tumor necrosis factor-alpha. Int Immunopharmacol 2005; 5: 1641-51.

35 Ding M, Yuan YJ. Study on the mechanisms of an extract of Salvia miltiorrhiza on the regulation of permeability of endothelial cells exposed to tumour necrosis factor-alpha. J Pharm Pharmacol 2007; 59: 1027-33.

36 Shi CS, Huang HC, Wu HL, Kuo CH, Chang BI, Shiao MS, et al. Salvianolic acid B modulates hemostasis properties of human umbilical vein endothelial cells. Thromb Res 2007; 119: 769-75.

37 Zhou Z, Liu Y, Miao AD, Wang SQ. Salvianolic acid B attenuates plasminogen activator inhibitor type 1 production in TNF-alpha treated human umbilical vein endothelial cells. J Cell Biochem 2005; 96: 109-16.

38 Chen YH, Lin SJ, Ku HH, Shiao MS, Lin FY, Chen JW, et al. Salvianolic acid $B$ attenuates VCAM-1 and ICAM-1 expression in TNF-alphatreated human aortic endothelial cells. J Cell Biochem 2001; 82: 512-21.
39 Xie LX, Durairajan SS, Lu JH, Liu CL, Kum WF, Wang Y, et al. The effect of salvianolic acid $B$ combined with laminar shear stress on TNF-alpha-stimulated adhesion molecule expression in human aortic endothelial cells. Clin Hemorheol Microcirc 2010; 44: 245-58.

40 Chen SC, Lin YL, Huang B, Wang DL, Cheng JJ. Salvianolic acid B suppresses IFN-gamma-induced JAK/STAT1 activation in endothelial cells. Thromb Res 2011; 128: 560-4.

41 Joe Y, Zheng M, Kim HJ, Kim S, Uddin MJ, Park C, et al. Salvianolic acid $B$ exerts vasoprotective effects through the modulation of heme Oxygenase-1 and arginase activities. J Pharmacol Exp Ther 2012; 341: 850-8.

42 Luo WB, Dong L, Wang YP. Effect of magnesium lithospermate $B$ on calcium and nitric oxide in endothelial cells upon hypoxia/ reoxygenation. Acta Pharmacol Sin 2002; 23: 930-6.

43 Luo WB, Wang YP. Magnesium lithospermate B inhibits hypoxiainduced calcium influx and nitric oxide release in endothelial cells. Acta Pharmacol Sin 2001; 22: 1135-42.

44 Pan C, Lou L, Huo Y, Singh G, Chen M, Zhang D, et al. Salvianolic acid $B$ and tanshinone IIA attenuate myocardial ischemia injury in mice by NO production through multiple pathways. Ther Adv Cardiovasc Dis 2011; 5: 99-111.

45 Sun A, Liu H, Wang S, Shi D, Xu L, Cheng Y, et al. Salvianolic acid B suppresses maturation of human monocyte-derived dendritic cells by activating PPARgamma. Br J Pharmacol 2011; 164: 2042-53.

46 Cheng CC, Yang SP, Lin WS, Ho LJ, Lai JH, Cheng SM, et al. Magnesium lithospermate $B$ mediates anti-inflammation targeting activator protein-1 and nuclear factor-kappa B signaling pathways in human peripheral T lymphocytes. Int Immunopharmacol 2012; 13: 354-61.

47 Sperl B, Seifert MH, Berg T. Natural product inhibitors of proteinprotein interactions mediated by Src-family $\mathrm{SH} 2$ domains. Bioorg Med Chem Lett 2009; 19: 3305-9.

48 Won J, Hur YG, Hur EM, Park SH, Kang MA, Choi Y, et al. Rosmarinic acid inhibits TCR-induced T cell activation and proliferation in an Lckdependent manner. Eur J Immunol 2003; 33: 870-9.

49 Wang L, Bao Y, Yang Y, Wu Y, Chen X, Si S, et al. Discovery of antagonists for human scavenger receptor CD36 via an ELISA-like high-throughput screening assay. J Biomol Screen 2010; 15: 23950.

50 Chen RJ, Jinn TR, Chen YC, Chung TY, Yang WH, Tzen JT. Active ingredients in Chinese medicines promoting blood circulation as $\mathrm{Na}^{+} / \mathrm{K}^{+}$-ATPase inhibitors. Acta Pharmacol Sin 2011; 32: 141-51.

51 Han B, Zhang X, Zhang Q, Zhao G, Wei J, Ma S, et al. Protective effects of salvianolate on microvascular flow in a porcine model of myocardial ischaemia and reperfusion. Arch Cardiovasc Dis 2011; 104: 313-24.

52 Jiang B, Wu W, Li M, Xu L, Sun K, Yang M, et al. Cardioprotection and matrix metalloproteinase- 9 regulation of salvianolic acids on myocardial infarction in rats. Planta Med 2009; 75: 1286-92.

53 Xu L, Deng Y, Feng L, Li D, Chen X, Ma C, et al. Cardio-protection of salvianolic acid $B$ through inhibition of apoptosis network. PLoS One 2011; 6: e24036.

54 Jiang B, Chen J, Xu L, Gao Z, Deng Y, Wang Y, et al. Salvianolic acid $B$ functioned as a competitive inhibitor of matrix metalloproteinase-9 and efficiently prevented cardiac remodeling. BMC Pharmacol 2010; 10: 10.

55 Wang Y, Xu F, Chen J, Shen X, Deng Y, Xu L, et al. Matrix metalloproteinase- 9 induces cardiac fibroblast migration, collagen and cytokine secretion: inhibition by salvianolic acid B from Salvia miltiorrhiza. Phytomedicine 2011; 19: 13-9.

56 Lu Y, Liu X, Liang X, Xiang L, Zhang W. Metabolomic strategy to study 
therapeutic and synergistic effects of tanshinone IIA, salvianolic acid $\mathrm{B}$ and ginsenoside $\mathrm{Rb1}$ in myocardial ischemia rats. J Ethnopharmacol 2011; 134: 45-9.

57 Lu Y, Zheng Y, Liu X, Liang X, Ngai S, Li T, et al. Metabolomic profiles of myocardial ischemia under treatment with salvianolic acid $B$. Chin Med 2012; 7: 6.

58 Wang W, Hu GY, Wang YP. Selective modulation of L-type calcium current by magnesium lithospermate $B$ in guinea-pig ventricular myocytes. Life Sci 2006; 78: 2989-97.

59 Au-Yeung KK, Zhu DY, O K, Siow YL. Inhibition of stress-activated protein kinase in the ischemic/reperfused heart: role of magnesium tanshinoate B in preventing apoptosis. Biochem Pharmacol 2001; 62: 483-93.

60 Wang J, Zhang Y, Guo LL, Wu GJ, Liu RH. Salvianolic acid B inhibits the TLR4-NFkappaB-TNFalpha pathway and attenuates neonatal rat cardiomyocyte injury induced by lipopolysaccharide. Chin J Integr Med 2011; 17: 775-9.

61 Jiang B, Zhang L, Li M, Wu W, Yang M, Wang J, et al. Salvianolic acids prevent acute doxorubicin cardiotoxicity in mice through suppression of oxidative stress. Food Chem Toxicol 2008; 46: 1510-5.

62 Han X, Liu JX, Li XZ. Salvianolic acid B inhibits autophagy and protects starving cardiac myocytes. Acta Pharmacol Sin 2011; 32: 38-44.

63 Chen L, Xuan $\sqcup$, Wang YP. Effects of magnesium lithospermate B and its analogues on $\mathrm{Ca}^{2+}$ homeostasis in cultured rat thoracic aorta vascular smooth muscle cells. Planta Med 2009; 75: 1573-9.

64 Lam FF, Yeung JH, Kwan YW, Chan KM, Or PM. Salvianolic acid B, an aqueous component of danshen (Salvia miltiorrhiza), relaxes rat coronary artery by inhibition of calcium channels. Eur J Pharmacol 2006; 553: 240-5.

65 Zhang $\mathrm{H}$, Zhang J, Zha R, Hu M, Wang Y. Magnesium lithospermate $\mathrm{B}$ decreases $\left[\mathrm{Ca}^{2+}\right]_{\mathrm{i}}$ in endothelial cells by inhibiting $\mathrm{K}^{+}$currents. Eur J Pharmacol 2011; 650: 285-9.

66 Lam FF, Seto SW, Kwan YW, Yeung JH, Chan P. Activation of the iberiotoxin-sensitive BKCa channels by salvianolic acid $B$ of the porcine coronary artery smooth muscle cells. Eur J Pharmacol 2006; 546: 28-35.

67 Zhang HF, Chen XQ, Hu GY, Wang YP. Magnesium lithospermate B dilates mesenteric arteries by activating BKCa currents and contracts arteries by inhibiting $K_{v}$ currents. Acta Pharmacol Sin 2010; 31: 665-70.

68 Yang Q, Wang S, Xie Y, Wang J, Li H, Zhou X, et al. Effect of salvianolic Acid $B$ and paeonol on blood lipid metabolism and hemorrheology in myocardial ischemia rabbits induced by pituitruin. Int J Mol Sci 2010; 11: 3696-704.

69 Wang F, Liu YY, Liu LY, Zeng QJ, Wang CS, Sun K, et al. The attenuation effect of 3,4-dihydroxy-phenyl lactic acid and salvianolic acid $B$ on venular thrombosis induced in rat mesentery by photochemical reaction. Clin Hemorheol Microcirc 2009; 42: 7-18.

70 Wang W, Wang YP, Sun WK, Xu YM, Xuan $\sqcup$. Effects of magnesium lithospermate $B$ on aggregation and $5-\mathrm{HT}$ release in rabbit washed platelets. Acta Pharmacol Sin 2000; 21: 859-63.

71 Li M, Zhao C, Wong RN, Goto S, Wang Z, Liao F. Inhibition of shearinduced platelet aggregation in rat by tetramethylpyrazine and salvianolic acid B. Clin Hemorheol Microcirc 2004; 31: 97-103.

72 Wu YP, Zhao XM, Pan SD, Guo de A, Wei R, Han JJ, et al. Salvianolic acid $B$ inhibits platelet adhesion under conditions of flow by a mechanism involving the collagen receptor alpha2beta1. Thromb Res 2008; 123: 298-305.

73 Ma C, Yao Y, Yue QX, Zhou XW, Yang PY, Wu WY, et al. Differential proteomic analysis of platelets suggested possible signal cascades network in platelets treated with salvianolic acid B. PLoS One 2011; 6: e14692.

74 Li XF, Wang YP. Depside salts from Salvia miltiorrhiza improve myocardial microperfusion in rats using laser Doppler flowmetry. Acta Pharmacol Sin 2007; 28: 789-95.

75 Kang DG, Oh H, Chung HT, Lee HS. Inhibition of angiotensin converting enzyme by lithospermic acid $B$ isolated from radix Salviae miltiorrhiza Bunge. Phytother Res 2003; 17: 917-20.

76 Lu B, Ye Z, Deng Y, Wu H, Feng J. MEK/ERK pathway mediates cytoprotection of salvianolic acid $B$ against oxidative stress-induced apoptosis in rat bone marrow stem cells. Cell Biol Int 2010; 34: 1063-8.

77 Chan SS, Chen JH, Hwang SM, Wang IJ, Li HJ, Lee RT, et al. Salvianolic acid B-vitamin C synergy in cardiac differentiation from embryonic stem cells. Biochem Biophys Res Commun 2009; 387: 723-8.

78 Chen YH, Du GH, Zhang JT. Salvianolic acid B protects brain against injuries caused by ischemia-reperfusion in rats. Acta Pharmacol Sin 2000; 21: 463-6.

79 Tzen JT, Jinn TR, Chen YC, Li FY, Cheng FC, Shi LS, et al. Magnesium lithospermate $\mathrm{B}$ possesses inhibitory activity on $\mathrm{Na}^{+}, \mathrm{K}^{+}$-ATPase and neuroprotective effects against ischemic stroke. Acta Pharmacol Sin 2007; 28: 609-15.

80 Li M, Zhao MQ, Kumar Durairajan SS, Xie LX, Zhang HX, Kum WF, et al. Protective effect of tetramethylpyrazine and salvianolic acid $B$ on apoptosis of rat cerebral microvascular endothelial cell under high shear stress. Clin Hemorheol Microcirc 2008; 38: 177-87.

81 Durairajan SS, Yuan Q, Xie L, Chan WS, Kum WF, Koo I, et al. Salvianolic acid $B$ inhibits Abeta fibril formation and disaggregates preformed fibrils and protects against Abeta-induced cytotoxicty. Neurochem Int 2008; 52: 741-50.

82 Kim DH, Park SJ, Kim JM, Jeon SJ, Cho YW, Son KH, et al. Cognitive dysfunctions induced by a cholinergic blockade and Abeta 25-35 peptide are attenuated by salvianolic acid B. Neuropharmacology 2011; 61: 1432-40.

83 Liu CL, Xie LX, Li M, Durairajan SS, Goto S, Huang JD. Salvianolic acid $B$ inhibits hydrogen peroxide-induced endothelial cell apoptosis through regulating PI3K/Akt signaling. PLoS One 2007; 2: e1321.

84 Zeng G, Tang T, Wu HJ, You WH, Luo JK, Lin Y, et al. Salvianolic acid B protects $\mathrm{SH}-\mathrm{SY} 5 \mathrm{Y}$ neuroblastoma cells from 1-methyl-4phenylpyridinium-induced apoptosis. Biol Pharm Bull 2010; 33: 1337-42.

85 Liu P, Hu YY, Liu C, Zhu DY, Xue HM, Xu ZQ, et al. Clinical observation of salvianolic acid $B$ in treatment of liver fibrosis in chronic hepatitis $B$. World J Gastroenterol 2002; 8: 679-85.

86 Lin YL, Wu CH, Luo MH, Huang YJ, Wang CN, Shiao MS, et al. In vitro protective effects of salvianolic acid B on primary hepatocytes and hepatic stellate cells. J Ethnopharmacol 2006; 105: 215-22.

87 Paik YH, Yoon YJ, Lee HC, Jung MK, Kang SH, Chung SI, et al. Antifibrotic effects of magnesium lithospermate $B$ on hepatic stellate cells and thioacetamide-induced cirrhotic rats. Exp Mol Med 2011; 43: 341-9.

88 Tsai MK, Lin YL, Huang YT. Effects of salvianolic acids on oxidative stress and hepatic fibrosis in rats. Toxicol Appl Pharmacol 2010; 242: 155-64.

89 Yao G, Xu L, Wu X, Yang J, Chen H. Preventive effects of salvianolic acid $B$ on transforming growth factor-beta1-induced epithelial-tomesenchymal transition of human kidney cells. Biol Pharm Bull 2009; 32: 882-6.

90 Pan RH, Xie FY, Chen HM, Xu LZ, Wu XC, Xu LL, et al. Salvianolic acid $B$ reverses the epithelial-to-mesenchymal transition of HK-2 cells 
that is induced by transforming growth factor-beta. Arch Pharm Res 2011; 34: 477-83.

91 Wang QL, Tao YY, Yuan JL, Shen L, Liu CH. Salvianolic acid B prevents epithelial-to-mesenchymal transition through the TGF-beta1 signal transduction pathway in vivo and in vitro. BMC Cell Biol 2010; 11: 31.

92 Chen CG, Wang YP. Magnesium lithospermate B ameliorates renal cortical microperfusion in rats. Acta Pharmacol Sin 2006; 27: $217-$ 22.

93 Xu M, Wang YP, Luo WB, Xuan $\sqcup$. Salvianolate inhibits proliferation and endothelin release in cultured rat mesangial cells. Acta Pharmacol Sin 2001; 22: 629-33.

94 Luo P, Tan Z, Zhang Z, Li H, Mo Z. Inhibitory effects of salvianolic acid $B$ on the high glucose-induced mesangial proliferation via NFkappaB-dependent pathway. Biol Pharm Bull 2008; 31: 1381-6.

95 Lee GT. Delayed treatment with lithospermate B attenuates experimental diabetic renal injury. J Am Soc Nephrol 2003; 14: 709-20.

96 Kang ES, Lee GT, Kim BS, Kim CH, Seo GH, Han SJ, et al. Lithospermic acid $\mathrm{B}$ ameliorates the development of diabetic nephropathy in OLETF rats. Eur J Pharmacol 2008; 579: 418-25.

97 Bulgakov VP, Inyushkina YV, Fedoreyev SA. Rosmarinic acid and its derivatives: biotechnology and applications. Crit Rev Biotechnol 2012; 32: 203-17.

98 Chu X, Ci X, He J, Jiang L, Wei M, Cao Q, et al. Effects of a natural prolyl oligopeptidase inhibitor, rosmarinic acid, on lipopolysaccharideinduced acute lung injury in mice. Molecules 2012; 17: 3586-98.

99 Kim DS, Kim HR, Woo ER, Hong ST, Chae HJ, Chae SW. Inhibitory effects of rosmarinic acid on adriamycin-induced apoptosis in H9c2 cardiac muscle cells by inhibiting reactive oxygen species and the activations of c-Jun N-terminal kinase and extracellular signalregulated kinase. Biochem Pharmacol 2005; 70: 1066-78.

100 Karthik D, Viswanathan P, Anuradha CV. Administration of rosmarinic acid reduces cardiopathology and blood pressure through inhibition of p22phox NADPH oxidase in fructose-fed hypertensive rats. J Cardiovasc Pharmacol 2011; 58: 514-21.

101 Fadel O, El Kirat K, Morandat S. The natural antioxidant rosmarinic acid spontaneously penetrates membranes to inhibit lipid peroxidation in situ. Biochim Biophys Acta 2011; 1808: 2973-80.

102 Hur YG, Suh CH, Kim S, Won J. Rosmarinic acid induces apoptosis of activated T cells from rheumatoid arthritis patients via mitochondrial pathway. J Clin Immunol 2007; 27: 36-45.

103 Lee HJ, Jeong YI, Lee TH, Jung ID, Lee JS, Lee CM, et al. Rosmarinic acid inhibits indoleamine 2,3-dioxygenase expression in murine dendritic cells. Biochem Pharmacol 2007; 73: 1412-21.

104 Alkam T, Nitta A, Mizoguchi H, Itoh A, Nabeshima T. A natural scavenger of peroxynitrites, rosmarinic acid, protects against impairment of memory induced by Abeta(25-35). Behav Brain Res 2007; 180: 139-45.

105 Jiang $\mathrm{H}$, Song $\mathrm{N}, \mathrm{Xu} \mathrm{H}$, Zhang S, Wang J, Xie J. Up-regulation of divalent metal transporter 1 in 6-hydroxydopamine intoxication is IRE/IRP dependent. Cell Res 2010; 20: 345-56.

106 Du T, Li L, Song N, Xie J, Jiang H. Rosmarinic acid antagonized 1-methyl-4-phenylpyridinium ( $\mathrm{MPP}^{+}$)-induced neurotoxicity in MES23.5 dopaminergic cells. Int J Toxicol 2010; 29: 625-33.

107 Wang J, Xu H, Jiang H, Du X, Sun P, Xie J. Neurorescue effect of rosmarinic acid on 6-hydroxydopamine-lesioned nigral dopamine neurons in rat model of Parkinson's disease. J Mol Neurosci 2012; 47: 113-9.

108 Jiang WL, Xu Y, Zhang SP, Hou J, Zhu HB. Effect of rosmarinic acid on experimental diabetic nephropathy. Basic Clin Pharmacol Toxicol 2012; 110: 390-5.

109 Yang MD, Chiang YM, Higashiyama R, Asahina K, Mann DA, Mann $J$, et al. Rosmarinic acid and baicalin epigenetically derepress peroxisomal proliferator-activated receptor gamma in hepatic stellate cells for their antifibrotic effect. Hepatology 2012; 55: 1271-81.

110 Liu X, Chen R, Shang Y, Jiao B, Huang C. Lithospermic acid as a novel xanthine oxidase inhibitor has anti-inflammatory and hypouricemic effects in rats. Chem Biol Interact 2008; 176: 137-42.

111 Chen L, Wang WY, Wang YP. Inhibitory effects of lithospermic acid on proliferation and migration of rat vascular smooth muscle cells. Acta Pharmacol Sin 2009; 30: 1245-52. 